\title{
Frequency dependence of dielectrophoretic fabrication of single-walled carbon nanotube field-effect transistors
}

\author{
Yousef Adeli Sadabad ${ }^{4} \cdot$ Amirreza Khodadadian $^{1,2} \cdot$ Kiarash Hosseini Istadeh $^{4} \cdot$ Marjan Hedayati $^{4}$. \\ Reza Kalantarinejad ${ }^{4}$. Clemens Heitzinger ${ }^{1,3}$
}

Received: 9 April 2020 / Accepted: 28 July 2020 / Published online: 10 August 2020

(c) The Author(s) 2020

\begin{abstract}
A new theoretical model for the dielectrophoretic (DEP) fabrication of single-walled carbon nanotubes (SWCNTs) is presented. A different frequency interval for the alignment of wide-energy-gap semiconductor SWCNTs is obtained, exhibiting a considerable difference from the prevalent model. Two specific models are study, namely the spherical model and the ellipsoid model, to estimate the frequency interval. Then, the DEP process is performed and the obtained frequencies (from the spherical and ellipsoid models) are used to align the SWCNTs. These empirical results confirm the theoretical predictions, representing a crucial step towards the realization of carbon nanotube field-effect transistors (CNT-FETs) via the DEP process based on the ellipsoid model.
\end{abstract}

Keywords Dielectrophoresis $\cdot$ Single-walled carbon nanotube $\cdot$ Spherical model $\cdot$ Ellipsoid model

\section{Introduction}

A graphene sheet rolled up into a cylinder forms a SWCNT, which can be either metallic or semiconducting depending on its geometrical structure. SWCNTs are a material with unique properties such as high tensile strength, high electrical/thermal conductivity, high elastic modulus, and ductility. All these properties mean that CNT-based products have great scientific importance, in addition to their great potential for use in technical applications such as thermal conduction enhancement, composites, filtration, sensors, and microelectronics.

Due to their unique electronic and mechanical properties, carbon nanotubes have attracted great attention for use in a

Amirreza Khodadadian

amirreza.khodadadian@tuwien.ac.at

1 Institute for Analysis and Scientific Computing, Vienna University of Technology (TU Wien), Wiedner Hauptstraße 8-10, 1040 Vienna, Austria

2 Institute of Applied Mathematics, Leibniz University Hannover, Welfengarten 1, 30167 Hanover, Germany

3 School of Mathematical and Statistical Sciences, Arizona State University, Tempe, AZ 85287, USA

4 Shezan Research and Innovation Center, No. 25, Innovation 2 St., Pardis TechPark, Tehran, Iran broad range of applications, especially in nanoelectronics and sensing applications [1-3]. Electronic devices fabricated using individual SWNTs have shown outstanding device performance, surpassing those of silicon. A critical step to obtain these practical devices is to deposit well-organized and highly aligned CNTs at desired locations. There are a number of different methods to align CNTs, such as chemical and biological patterning, Langmuir-Blodgett assembly, etc. The interested reader can refer to Refs. [4-9]. However, these methods have their shortcomings and limitations, such as intensive preparation processes or the requirement for assisting materials with special properties. Also, most of these techniques lack precise control over the positioning and orientation of individual SWNTs. Therefore, they may not be appropriate for scaled-up fabrication of individual SWCNT devices.

The dielectrophoresis technique was first adopted by Pohl [10] and involves the motion of suspended particles relative to the solvent due to polarization forces produced by an inhomogeneous electric field. This technique is simple but versatile and can be conducted at room temperature using low voltages, and thus can be applied to align CNTs between electrodes with high yield $[11,12]$. Several parameters have great importance in the dielectrophoresis technique, such as the alternating voltage (AC) amplitude, the frequency of 
the applied voltage, the deposition time, the geometry of the electrodes, and the concentration of the solution.

In Ref. [13], multiwalled carbon nanotubes with a variety of sizes were assembled onto electrodes using alternatingcurrent electric fields by dielectrophoresis. Assembly of CNTs between electrodes using a combination of optically induced dielectrophoresis and dielectrophoresis forces was performed in Ref. [14]. A different study on the directed assembly of CNTs and graphene in an alternating current (AC) electric field can be found in Ref. [15]. The authors of Ref. [16] used simulation results to clarify the role of the medium in the motion of CNTs during such alignment using a DEP process prior to actual alignment.

One of the most important problems when using this technique to align CNTs between electrodes is that metallic carbon nanotubes are always present along with semiconducting CNTs in the solution, so choosing appropriate values for the parameters in order to absorb only semiconductor CNTs is an important consideration.

The work presented herein focuses on the frequency of the applied voltage, revealing that the use of a suitable theoretical model for this technique can identify an appropriate frequency (one of the important parameters) in this process to absorb and align semiconductor CNTs between electrodes and consequently achieve $p$-type CNT-FET behavior of the fabricated device.

A prevalent model for DEP, based on a chain of spherical particles along the CNT axis [17], is first derived and demonstrated, then a new model based on a prolate ellipsoid is applied. Using this new model, the frequency of the applied voltage can be chosen properly, as different values are calculated to absorb semiconductor CNTs compared with the previous model. This new theoretical result reveals the reason why devices show metallic or low-bandgap semiconductor behavior, as our experiment suggested. On the other hand, it provides pertinent values in terms of the frequency to yield semiconducting characteristics. The calculated results are consistent with experiment, whereas previously calculated parameters at a particular frequency led to mostly metallic or quasimetallic behavior. The theoretical results can be extended to solve different novel problems in electrochemistry, e.g., [18-21], modern physics [21-26], as well as the simulation of silicon nanowire sensors [27-31], field-effect transistors [32,33], and ion channels [34].

The remainder of this manuscript is organized as follows: Section 2 explains the theoretical work and obtains the frequency interval for the spherical model. Section 3 describes the ellipsoid model and estimates the corresponding frequency interval. In Sect. 4, the frequencies obtained (according to the spherical and ellipsoid models) are employed, and the $I-V$ characteristics of the fabricated devices are plotted to identify the type (metallic or semiconductor) of aligned SWCNTs. Finally, conclusions are drawn in Sect. 5.

\section{The theoretical work}

The basic idea behind the theory of dielectrophoresis is that a dielectric particle in a nonuniform electric field experiences a net force that depends on the electric field and the effective dipole moment of the particle generated thereby.

Dielectrophoresis is a phenomenon in which a force is exerted on a dielectric particle when suspended in a nonuniform electric field. The polarization of the particles by a nonuniform electric field makes it possible to exert a DEP force even on uncharged particles. All polarizable particles exhibit the dielectrophoresis phenomenon in the presence of an electric field. The electrical properties of the particles and medium, the shape and size of the particles, the strength of the electric field, and the frequency of the applied field are the main parameters that determine the strength of the exerted force.

The dipole consists of equal and opposite charges $(+q$ and $-q$ ) separated by a distance vector $\boldsymbol{d}$, and is located in an electric field $\boldsymbol{E}$. If the electric field is nonuniform, then, in general, the two charges $(+q$ and $-q)$ will experience different values of the vector field $\boldsymbol{E}$ and the dipole will experience a net force. The total force on the dipole is

$\boldsymbol{F}=q \boldsymbol{E}(\boldsymbol{t}+\boldsymbol{d})-q \boldsymbol{E}(\boldsymbol{r})$,

where $\boldsymbol{r}$ is the position of the particle with charge $-q$. The first-order approximation (dielectrophoretic approximation) is given by

$\boldsymbol{F}_{\text {dipole }}=(q \boldsymbol{d} \cdot \nabla) \boldsymbol{E}(\boldsymbol{r})$.

Therefore, to calculate the force on a dipole given the electric field, the effective moment of the dielectric particle must first be calculated. In the current work, SWCNTs suspended in a medium are modeled. The first model, which is prevalently used, considers the CNTs as a chain of spherical particles, so we first present the main formula for the effective dipole moment of a spherical dielectric particle in an external electric field and then proceed to the new model.

For a spherical dielectric particle in an external electric field [35], consider an electric field in the $\hat{z}$ direction and solve Laplace's equation to yield the potential functions inside and outside the sphere:

$\phi_{1}(r, \theta)=-E_{0} r \cos \theta+\left(\frac{\varepsilon_{\mathrm{p}}-\varepsilon_{\mathrm{m}}}{\varepsilon_{\mathrm{p}}+2 \varepsilon_{\mathrm{m}}} R^{3} E_{0}\right) \frac{\cos \theta}{r^{2}}, \quad r \geq R$,

$\phi_{2}(r, \theta)=-\left(\frac{3 \varepsilon_{\mathrm{m}}}{\varepsilon_{\mathrm{p}}+2 \varepsilon_{\mathrm{m}}} E_{0}\right) r \cos \theta, \quad r<R$,

where $\varepsilon_{\mathrm{m}}$ is the permittivity of the medium and $\varepsilon_{\mathrm{p}}$ is that of particle, while the second term indicates the dipole. Furthermore, note that the electrostatic potential $\phi_{\text {dipole }}$ due to 
a point dipole of moment $\rho_{\text {eff }}$ in a dielectric medium of permittivity $\varepsilon_{\mathrm{m}}$ is [35]

$\phi_{\text {dipole }}=\frac{\rho_{\text {eff }} \cos \theta}{4 \pi \varepsilon_{\mathrm{m}} r^{2}}$.

Comparing Eq. (5) and Eq. (3), it can be concluded that

$\rho_{\text {eff }}=4 \pi \varepsilon_{\mathrm{m}} \frac{\varepsilon_{\mathrm{p}}-\varepsilon_{\mathrm{m}}}{\varepsilon_{\mathrm{p}}+2 \varepsilon_{\mathrm{m}}} R^{3} E_{0}$,

consequently for the special case of a homogeneous dielectric sphere, the expression for the effective dipole moment is

$\rho_{\text {eff }}=4 \pi \varepsilon_{\mathrm{m}} \mathcal{K} R^{3} E_{0}$.

Here, $\mathcal{K}$, known as the Clausius-Mossotti function [36], provides a measure of the strength of the effective polarization of a spherical particle as a function of $\varepsilon_{\mathrm{p}}$ and $\varepsilon_{\mathrm{m}}$

$\mathcal{K}\left(\varepsilon_{\mathrm{p}}, \varepsilon_{\mathrm{m}}\right)=\frac{\varepsilon_{\mathrm{p}}-\varepsilon_{\mathrm{m}}}{\varepsilon_{\mathrm{p}}+2 \varepsilon_{\mathrm{m}}}$.

When $\varepsilon_{\mathrm{p}}>\varepsilon_{\mathrm{m}}$, then $\mathcal{K}$ and the effective dipole moment $\rho_{\text {eff }}$ is collinear with the electric field vector, but when $\varepsilon_{\mathrm{p}}<\varepsilon_{\mathrm{m}}$, the two vectors are antiparallel.

Now consider an AC electric field and assume that the sphere and medium have finite conductivities.

$\boldsymbol{E}(t)=E_{0} \exp (\mathrm{i} \omega t) \hat{z}$.

The solution is the same as Eqs. (3) and (4), except that the coefficients are now complex and a more general expression for the complex effective moment results:

$\rho_{\text {eff }}=4 \pi \varepsilon_{\mathrm{m}} \bar{K} R^{3} \boldsymbol{E}(t)$.

The Clausius-Mossotti factor now becomes a function of the complex permittivities, containing magnitude and phase information about the effective dipole moment. Consider complex coordinates as

$\overline{\varepsilon_{\mathrm{m}}}=\varepsilon_{\mathrm{m}}-\mathrm{i} \frac{\sigma_{\mathrm{m}}}{\omega}, \quad \overline{\varepsilon_{\mathrm{p}}}=\varepsilon_{\mathrm{p}}-\mathrm{i} \frac{\sigma_{\mathrm{p}}}{\omega}$,

where $\sigma_{\mathrm{m}}$ and $\sigma_{\mathrm{p}}$ indicate the conductivity of medium and the particle, respectively. Such ohmic, dispersive behavior is a consequence of the finite time required to build up the surface charge $\sigma$ at the interface.

Assuming the spatial variation for the electric field from Eqs. (10) and (2), the time-averaged force is

$\boldsymbol{F}_{\text {DEP }}=\frac{1}{2} \mathcal{R}\left\{\left(\rho_{\text {eff }} \cdot \nabla\right) \overline{\boldsymbol{E}}(t, x)\right\}=\pi \varepsilon_{\mathrm{m}} \mathcal{R}\{K\} R^{3} \nabla\left(E^{2}\right)$,

where $\overline{\boldsymbol{E}}(t, x)$ indicates the complex conjugate of $\boldsymbol{E}(t, x)$ and the real and imaginary parts are given by [36]
$\mathcal{R}\left\{\mathcal{K}\left(\overline{\varepsilon_{\mathrm{p}}}, \bar{\varepsilon}_{\mathrm{m}}^{-}\right)\right\}=\frac{\left(\varepsilon_{\mathrm{p}}-\varepsilon_{\mathrm{m}}\right)\left(\varepsilon_{\mathrm{p}}+2 \varepsilon_{\mathrm{m}}\right)+\left(\frac{1}{\omega}\right)^{2}\left(\sigma_{\mathrm{p}}-\sigma_{\mathrm{m}}\right)\left(\sigma_{\mathrm{p}}+2 \sigma_{\mathrm{m}}\right)}{\left(\varepsilon_{\mathrm{p}}+2 \varepsilon_{\mathrm{m}}\right)^{2}+\left(\frac{1}{\omega}\right)^{2}\left(\sigma_{\mathrm{p}}+2 \sigma_{\mathrm{m}}\right)^{2}}$

$\mathcal{I}\left\{\mathcal{K}\left(\overline{\varepsilon_{\mathrm{p}}}, \varepsilon_{\mathrm{m}}^{-}\right)=\frac{3}{\omega} \frac{\varepsilon_{\mathrm{p}} \sigma_{\mathrm{m}}-\varepsilon_{\mathrm{m}} \sigma_{\mathrm{p}}}{\left.\left(\varepsilon_{\mathrm{p}}+2 \varepsilon_{\mathrm{m}}\right)^{2}+\frac{1}{\omega}\right)^{2}\left(\sigma_{\mathrm{p}}+2 \sigma_{\mathrm{m}}\right)^{2}}\right.$.

Also, the relevant relaxation time constant in this case is $\tau=\frac{\varepsilon_{\mathrm{p}}+2 \varepsilon_{\mathrm{m}}}{\sigma_{\mathrm{p}}+2 \sigma_{3}}$, i.e., the reciprocal of the frequency for which $\mathcal{R}\{\mathcal{K}\}$ is maximum. The important point about this constant is that, whenever the relaxation time constant is longer than the reciprocal of the applied frequency, the particle will respond to the time-averaged force and torque, i.e., $\tau>\frac{1}{\omega_{\text {applied voltage }}}$.

From Eq. (12) it is clear that, to absorb CNTs onto the region of strong electric field (downward between the electrodes), the term $\mathcal{R}\{\mathcal{K}\}$ should be positive. Assuming that $\varepsilon_{\mathrm{p}} \leq \varepsilon_{\mathrm{m}}$ and $\sigma_{\mathrm{p}} \geq \sigma_{\mathrm{m}}$ (wide-energy-gap semiconductor SWCNTs in aqueous solution), we have the following formula for the zero-force frequency (the frequency at which the particle experiences no time-averaged force):

$\omega\left(\mathcal{R}\left\{\mathcal{K}\left(\overline{\varepsilon_{\mathrm{p}}}, \overline{\varepsilon_{\mathrm{m}}}\right)\right\}=0\right)=\sqrt{\frac{\left(\sigma_{\mathrm{p}}-\sigma_{\mathrm{m}}\right)\left(\sigma_{\mathrm{p}}+2 \sigma_{\mathrm{m}}\right)}{\left|\left(\varepsilon_{\mathrm{p}}-\varepsilon_{\mathrm{m}}\right)\left(\varepsilon_{\mathrm{p}}+2 \varepsilon_{\mathrm{m}}\right)\right|}}$.

The key point is obtaining the below frequency that we desire from the direction of force to align nanotubes between electrodes.

Considering SWCNTs solved in deionized water, Eq. 15 is used to calculate the frequency of zero force, where $\varepsilon_{\mathrm{p}}=30 \varepsilon_{0}, \quad \sigma_{\mathrm{p}}=10^{-2} \mathrm{~S} / \mathrm{m}, \quad \varepsilon_{\mathrm{m}}=70 \varepsilon_{0}$, and $\sigma_{\mathrm{m}}=5.5 \times 10^{-6} \mathrm{~S} / \mathrm{m}$ are assumed. Here, $\varepsilon_{\mathrm{p}} \approx \varepsilon_{0}$ for largegap semiconductor SWNTs, with larger values for smallgap and metallic tubes. It can therefore be concluded that

$\omega\left(\mathcal{R}\left\{\mathcal{K}\left(\overline{\varepsilon_{\mathrm{p}}}, \overline{\varepsilon_{\mathrm{m}}}\right)\right\}=0\right) \cong 13.7 \mathrm{MHz}, \quad \frac{1}{\tau} \cong 6.6 \mathrm{MHz}$.

So, to attract an SWCNT with given parameter values, the frequency of the applied field should lie in the interval

$6.6 \mathrm{MHz}<\omega<13.7 \mathrm{MHz}$.

An important characteristic of Eq. (15) is that $\omega\left(\mathcal{R}\left\{\mathcal{K}\left(\overline{\varepsilon_{\mathrm{p}}}, \overline{\varepsilon_{\mathrm{m}}}\right)\right\}=0\right)$ increases with $\sigma_{\mathrm{p}}$

$\frac{\partial}{\partial \sigma_{\mathrm{p}}} \omega^{2}\left(\mathcal{R}\left\{\mathcal{K}\left(\overline{\varepsilon_{\mathrm{p}}}, \bar{\varepsilon}_{\mathrm{m}}^{-}\right)\right\}=0\right)=\frac{\left(2 \sigma_{\mathrm{p}}+\sigma_{\mathrm{m}}\right)}{\left|\left(\varepsilon_{\mathrm{p}}-\varepsilon_{\mathrm{m}}\right)\left(\varepsilon_{\mathrm{p}}+2 \varepsilon_{\mathrm{m}}\right)\right|}>0$.

This means that the frequency of zero force shifts to the right with increasing conductivity of the particle, thus to absorb SWCNTs with lower conductivity (wider bandgap), lower frequencies should be used, and vice versa. Figure 1 shows 
Fig. 1 The frequency as a function of $\sigma_{\mathrm{p}}$

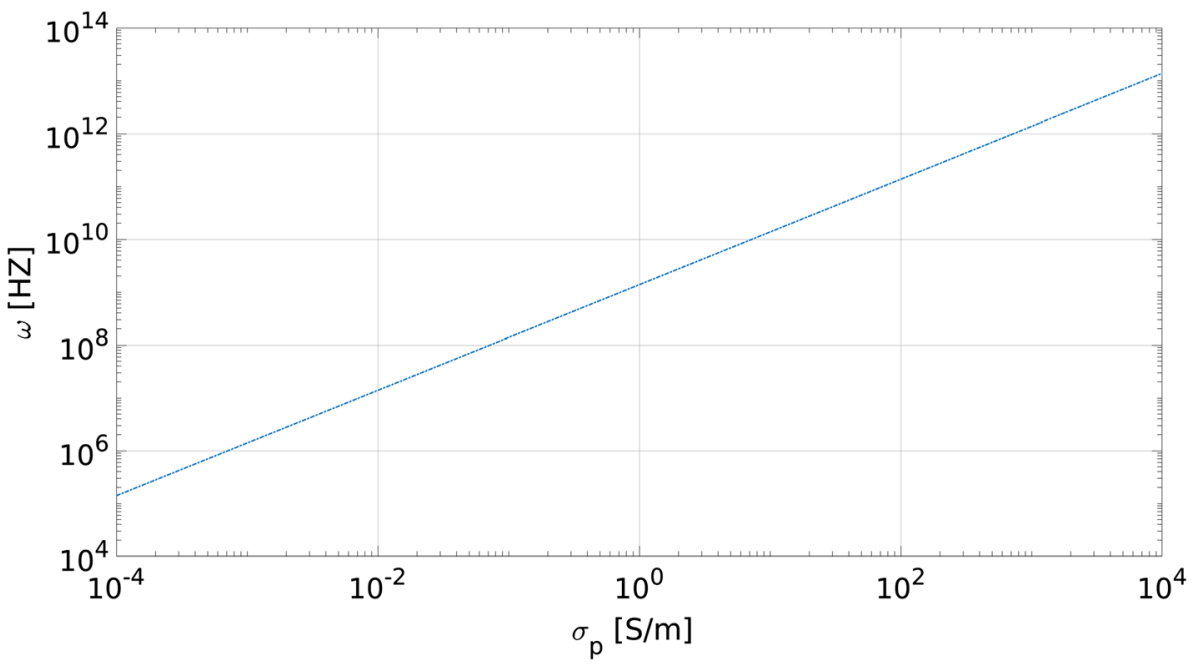

the dependence of the frequency on $\sigma_{\mathrm{p}}$ between $10^{-4} \mathrm{~S} / \mathrm{m}$ and $10^{4} \mathrm{~S} / \mathrm{m}$.

\section{The ellipsoid particle model of the CNTs}

A more realistic model is now applied, based on prolate ellipsoid, to identify the correct interval for the frequency of the applied voltage. In Ref. [37], a model based on ellipsoid particles in an electric field was introduced. The model predicts the potential at the ellipsoid's surface leading to the induced dipole moment. The aim of this section is to introduce an ellipsoid model for nanotubes to determine the proper frequency interval for the applied electric field to absorb semiconductor nanotubes and compare the result with the prevalent model (spherical model). In other words, the main goal is to show that the ellipsoid model is better suited to model cylindrically shaped nanotubes, and that by using this model a better estimate of the frequency interval of the applied electric field to absorb semiconductor carbon nanotubes can be obtained.

The problem of an ellipsoid in an electric field can be solved by introducing elliptical coordinates [38]. Note that, in the case of an SWCNT, there are different permittivities and conductivities in different directions. In the case of semiconductors, the longitudinal polarizability factor of the SWCNT is typically more than ten times greater its transverse polarizability [39]. Furthermore, the dipoles generated in the middle part cancel each other pairwise, so the generated dipole has a high aspect ratio, i.e., length to radius ratio.

Figure 2 illustrates the general idea of the ellipsoid model for the SWCNT dipole. In an external electric field, all the atoms are polarized, and those atoms located near each other overlap, resulting in two main centers with opposite charge:

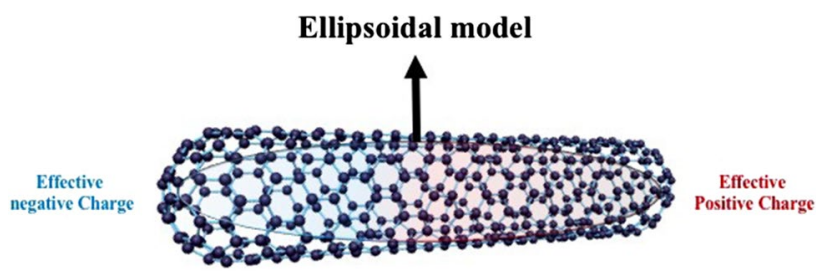

Fig. 2 The polarization of a SWCNT

a positive one on the leftmost side and a negative one on the rightmost side, giving a dipole whose characteristic length is the length of the SWCNT.

The resulting equation for the induced dipole moment in direction $n$ is

$\rho_{\mathrm{eff}-n}=\frac{4 \pi a_{1} a_{2} a_{3}}{3} \varepsilon_{\mathrm{m}}\left[\frac{\bar{\varepsilon}_{\mathrm{p}-n}-\bar{\varepsilon}_{\mathrm{m}}}{\bar{\varepsilon}_{\mathrm{m}}+A_{n}\left(\bar{\varepsilon}_{\mathrm{p}-n}-\bar{\varepsilon}_{\mathrm{m}}\right)}\right] E_{n}$,

where $A_{n}$ is the depolarization factor for axis $n$

$A_{n}=\frac{a_{1} a_{2} a_{3}}{2} \int_{0}^{\infty} \frac{\mathrm{d} x}{\left(x+a_{n}^{2}\right) \sqrt{\left(x+a_{1}^{2}\right)\left(x+a_{2}^{2}\right)\left(x+a_{3}^{2}\right)}}$

where $a_{1}, a_{2}$, and $a_{3}$ are the half-lengths along the axes and $\bar{\varepsilon}_{\mathrm{p}-n}$ is the complex permittivity in direction $n$. Comparing Eqs. (10) to (19), it can be concluded that, in this case, the Clausius-Mossotti factor becomes

$\mathcal{K}_{n}\left(\bar{\varepsilon}_{\mathrm{p}}, \bar{\varepsilon}_{\mathrm{m}}, a_{1}, a_{2}, a_{3}\right)=\frac{1}{3} \frac{\bar{\varepsilon}_{\mathrm{p}-n}-\bar{\varepsilon}_{\mathrm{m}}}{\bar{\varepsilon}_{\mathrm{m}}+A_{n}\left(\bar{\varepsilon}_{\mathrm{p}-n}-\bar{\varepsilon}_{\mathrm{m}}\right)}$.

If $a_{1}=a_{2}=a_{3}$, all three $A_{n}$ are $\frac{1}{3}$, so we get the same result as for the Clausius-Mossotti factor for the sphere. If $a_{1} \gg a_{2}=a_{3}$, the $A_{n}$ can be calculated as 
$A_{1}=\frac{a_{1} a_{2} a_{3}}{2} \int_{0}^{\infty} \frac{\mathrm{d} x}{\left(x+a_{2}^{2}\right)\left(x+a_{1}^{2}\right)^{\frac{3}{2}}}=\frac{1}{2} \frac{1-e^{2}}{e^{3}}\left[\ln \frac{1+e}{1-e}-2 e\right]$,

where $e$ is the eccentricity, defined as

$e=\sqrt{1-\left(\frac{a_{2}}{a_{1}}\right)^{2}} \approx 1-\frac{1}{2}\left(\frac{a_{2}}{a_{1}}\right)^{2}+\frac{1}{8}\left(\frac{a_{2}}{a_{1}}\right)^{4}+\cdots$.

For very long, needle-shaped ellipsoid, the following approximation applies for $A_{1}$ :

$A_{1} \approx\left(\frac{a_{2}}{a_{1}}\right)^{2}\left(\ln \frac{2 a_{1}}{a_{2}}-1\right)$.

For $A_{2}$ and $A_{3}$,

$$
\begin{aligned}
A_{2}= & A_{3}=\frac{a_{1} a_{2}^{2}}{2} \int_{0}^{\infty} \frac{\mathrm{d} x}{\left(x+a_{2}^{2}\right) \sqrt{\left(x+a_{1}^{2}\right)}} \\
= & \frac{1}{2 e^{2}}-\frac{1}{2} \frac{1-e^{2}}{4 e^{3}}\left[\ln \frac{1+e}{1-e}\right] .
\end{aligned}
$$

In the case of a SWCNT with diameter-to-length ratio of $\frac{a_{2}}{a_{1}}$ (which is relevant in our case), then $A_{1} \sim 6.6 \times 10^{-6}$ and $A_{2}=A_{3} \sim \frac{1}{2}$. Equations (19) and (2) then yield the following formula for the time-averaged force exerted on a SWCNT in this model:

$$
\begin{aligned}
& \boldsymbol{F}_{\text {ellipsoidal dipole }}=\frac{2 \pi a_{1} a_{2} a_{3}}{3} \varepsilon_{\mathrm{m}} \\
& \qquad\left(\mathcal{R}\left\{\frac{\bar{\varepsilon}_{\mathrm{p}-n}-\bar{\varepsilon}_{\mathrm{m}}}{\bar{\varepsilon}_{\mathrm{m}}+A_{n}\left(\bar{\varepsilon}_{\mathrm{p}-n}-\bar{\varepsilon}_{\mathrm{m}}\right)} E_{n} \frac{\partial}{\partial x_{n}}\right\}\right) \boldsymbol{E} .
\end{aligned}
$$

As before, we need the real and imaginary parts of the Clausius-Mossotti factor, obtained from Eq. (26) as
The relaxation time constant for each direction in this case is then

$\tau_{n}=\frac{A_{n} \varepsilon_{\mathrm{p}-n}+\left(1-A_{n}\right) \varepsilon_{\mathrm{m}}}{A_{n} \sigma_{\mathrm{p}-n}+\left(1-A_{n}\right) \sigma_{\mathrm{m}}}$.

The point here is that an ellipsoid particle in an electric field always aligns itself with its longest axes along the direction of the electric field, so in this case, the dominant term is $\tau_{1}$.

In the case of a long SWCNT with a high aspect ratio, the following approximate formula for the force experienced by the SWCNT applies:

$\boldsymbol{F}_{\text {ellipsoidal dipole }}=\frac{\pi a_{1} a_{2} a_{3}}{3} \varepsilon_{\mathrm{m}} \mathcal{R}\left\{\frac{\bar{\varepsilon}_{\mathrm{pl}}-\bar{\varepsilon}_{\mathrm{m}}}{\bar{\varepsilon}_{\mathrm{m}}+A_{1}\left(\bar{\varepsilon}_{\mathrm{pl}}-\bar{\varepsilon}_{\mathrm{m}}\right)}\right\} \nabla E^{2}$,

where $\bar{\varepsilon}_{\mathrm{pl}}$ is the longitudinal complex permittivity. The frequency dependence of the force is present in the $\mathcal{R}\left\{\frac{\bar{\varepsilon}_{\mathrm{pl}}-\bar{\varepsilon}_{\mathrm{m}}}{\left.\bar{\varepsilon}_{\mathrm{m}}+A_{1} \bar{\varepsilon}_{\mathrm{pl}}-\bar{\varepsilon}_{\mathrm{m}}\right)}\right\}$ term. With this approximation, the following range of applied frequency is obtained:

$\frac{1}{\tau_{1}}<\omega<\omega\left(\mathcal{R}\left(\mathcal{K}_{1}\left(\bar{\varepsilon}_{\mathrm{pl}}, \bar{\varepsilon}_{\mathrm{m}}, a_{1}, a_{2}, a_{3}\right)=0\right)\right)$,

where the right-hand side indicates

$$
\begin{aligned}
\omega & \left(\mathcal{R}\left(\mathcal{K}_{1}\left(\bar{\varepsilon}_{\mathrm{pl}}, \bar{\varepsilon}_{\mathrm{m}}, a_{1}, a_{2}, a_{3}\right)=0\right)\right) \\
& =\sqrt{\frac{\left(\sigma_{\mathrm{pl}}-\sigma_{\mathrm{m}}\right)\left(A_{1} \sigma_{\mathrm{pl}}+\left(1-A_{1}\right) \sigma_{\mathrm{m}}\right)}{\left(\varepsilon_{\mathrm{pl}}-\varepsilon_{\mathrm{m}}\right)\left(A_{1} \varepsilon_{\mathrm{pl}}+\left(1-A_{1}\right) \varepsilon_{\mathrm{m}}\right)}}
\end{aligned}
$$

To estimate the frequency, the effective parameters summarized in Table 1 are applied, yielding

$9 \mathrm{KHz}<\omega<500 \mathrm{kHz}$.

$$
\begin{aligned}
\mathcal{R}\left(\mathcal{K}_{n}\left(\bar{\varepsilon}_{\mathrm{p}}, \bar{\varepsilon}_{\mathrm{m}}, a_{1}, a_{2}, a_{3}\right)\right) \\
=\frac{1}{3} \frac{\left(\varepsilon_{\mathrm{p}-n}-\varepsilon_{\mathrm{m}}\right)\left(A_{n} \varepsilon_{\mathrm{p}-n}+\left(1-A_{n}\right) \varepsilon_{\mathrm{m}}\right)+\left(\frac{1}{\omega}\right)^{2}\left(\sigma_{\mathrm{p}-n}-\sigma_{\mathrm{m}}\right)\left(A_{n} \sigma_{\mathrm{p}-n}+\left(1-A_{n}\right) \sigma_{\mathrm{m}}\right)}{\left(A_{n} \varepsilon_{\mathrm{p}-n}+\left(1-A_{n}\right) \varepsilon_{\mathrm{m}}\right)^{2}+\left(\frac{1}{\omega}\right)^{2}\left(A_{n} \sigma_{\mathrm{p}-n}+\left(1-A_{n}\right) \sigma_{\mathrm{m}}\right)^{2}}
\end{aligned}
$$

Here as in Eq. 18, with increasing electrical conductivity of the SWCNTs, the frequency of zero force shifts to the right while the relaxation time constant increases, so to absorb SWCNTs with higher electrical conductivity higher frequencies should be used, which results in absorption of more metal SWCNTs, so fabricated devices will show metallic
Table 1 The parameter values used to obtain the frequency

\begin{tabular}{llllllll}
\hline Parameter & $\varepsilon_{\mathrm{pl}}$ & $\varepsilon_{\mathrm{pt}}$ & $\sigma_{\mathrm{pl}}$ & $\sigma_{\mathrm{pt}}$ & $\varepsilon_{\mathrm{m}}$ & $\sigma_{\mathrm{m}}$ & $A_{1}$ \\
\hline Value & $30 \varepsilon_{0}$ & $8 \varepsilon_{0}$ & $10^{-2} \mathrm{~S} / \mathrm{m}$ & 0 & $70 \varepsilon_{0}$ & $5.5 \times 10^{-6} \mathrm{~S} / \mathrm{m}$ & $6.6 \times 10^{-6}$ \\
\hline
\end{tabular}


behavior. Moreover, because of the presence of other forces that result in different motions such as electrothermal motion or Brownian motion, the frequency should be chosen close to the lower limit to achieve a strong DEP force and remain close to the stable polarization.

An important point here is that the transverse conductivity of a SWCNT is much lower than its longitudinal conductivity and can thus be assumed to be zero. Figure 3 shows that variation in this assumption does not have a considerable effect on the real and imaginary parts of the Clausius-Mossotti factor. According to Fig. 3, the variation in $\mathcal{R}$ is restricted to the interval $(-0.6,0.7)$ while that of $\mathcal{I}$ is restricted to the interval $(-0.3,0.1)$, so overall $\mathcal{R}$ is restricted to $(-1.3,1.2)$ and $\mathcal{I}$ to $(-0.3,0.2)$, which are not comparable to the longitudinal factors at the relevant frequency.

The point here is that, because of the presence of other forces which result in different motions such as electrothermal motion or Brownian motion, the frequency should be chosen near to the lower limit to achieve a stronger DEP force and remain near to the stable polarization.

\section{Experimental results}

To identify the type (metallic or semiconductor) of aligned SWCNTs when using the two different applied frequencies obtained based on the two models, the $I-V$ characteristics of fabricated devices are plotted. It is known that, for metallic SWCNTs, a straight line is observed in the $I-V$ plane, whereas for semiconductor SWCNTs, we expect a nonlinear relationship between the current and applied voltage. Indeed, for fabricated devices with a dominant number of semiconductor SWCNTs, with increasing voltage more and more holes participate in the current generation, and we would see an exponential $I-V$ characteristic.

The SWCNTs used in the experiment are in the form of an aqueous solution and purchased from Nanointegris (http://www.nanointegris.com/). The specification for the SWNT solution is: diameter range from 1.2 to $1.7 \mathrm{~nm}$, length range from $300 \mathrm{~nm}$ and $5 \mu \mathrm{m}$, metal catalyst impurity $<1 \%$, amorphous carbon impurity $1-5 \%$, electronic enrichment 98\% semiconductor SWCNT. We diluted the solution to $100 \mathrm{ng} / \mathrm{ml}$ and used it in the DEP process.

For the source and drain electrodes, we used two kinds of electrodes: one with a 2- $\mu \mathrm{m}$ gap and another with a 1- $\mu \mathrm{m}$ gap, both having a width of $3 \mu \mathrm{m}$ at the end tip. The electrodes are shown in Fig. 4.

We performed the DEP process, and in order to align the SWCNTs between the electrodes, applied a voltage with an amplitude of $6 \mathrm{~V}$ for the electrodes with a $2-\mu \mathrm{m}$ gap and $5 \mathrm{~V}$ for the $1-\mu \mathrm{m}$ gap

The aim is now to use the obtained theoretical results (the estimated frequencies) to align the SWCNTs. The prevalent (spherical) model suggests that, in order to absorb semiconductor carbon nanotubes with the given parameters, an AC electric field with a frequency near $7 \mathrm{MHz}$ should be used. Meanwhile, the second (ellipsoid) model suggests the use of a frequency as low as $10 \mathrm{kHz}$. In the experiment, we used $50 \mathrm{kHz}$.

The duration of the DEP process in all the experiments was $20 \mathrm{~s}$. Figures 5 and 6 show the $I-V$ curves of the SWCNTs with a gap between the electrodes of 1 and $2 \mu \mathrm{m}$, as a function of the source-to-drain voltage. Here, the curves are related to the different gate voltage. The high linearity of the curves shows that most of the deposited CNT(s) were metallic (low-bandgap semiconductor) [40]. Figures 7 and 8 show the current for several $V_{\mathrm{g}}$ and $V_{\mathrm{SD}}$ voltages in two
Fig. 3 The real and imaginary parts of the CM factor versus the transverse conductivity at a frequency of $10 \mathrm{kHz}$

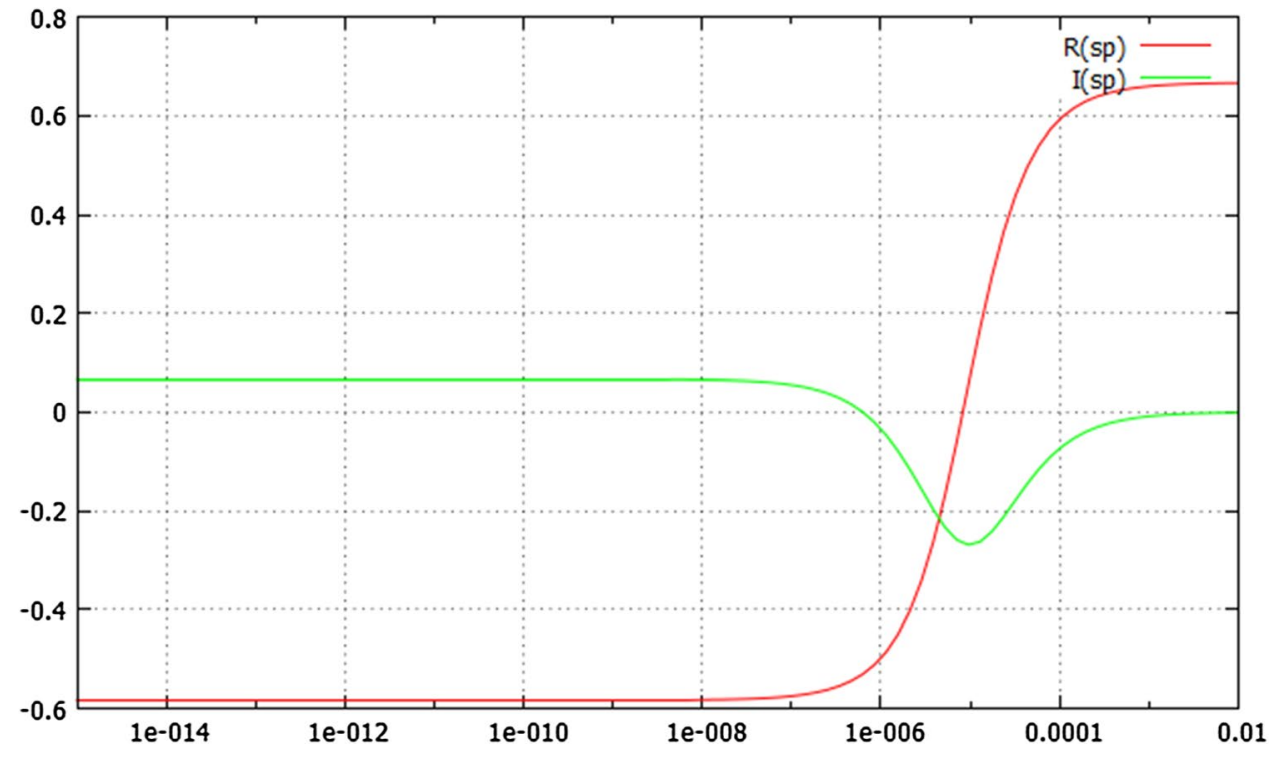


Fig. 4 The electrodes with a $2-\mu \mathrm{m}$ gap (top) and $1-\mu \mathrm{m}$ gap (bottom)
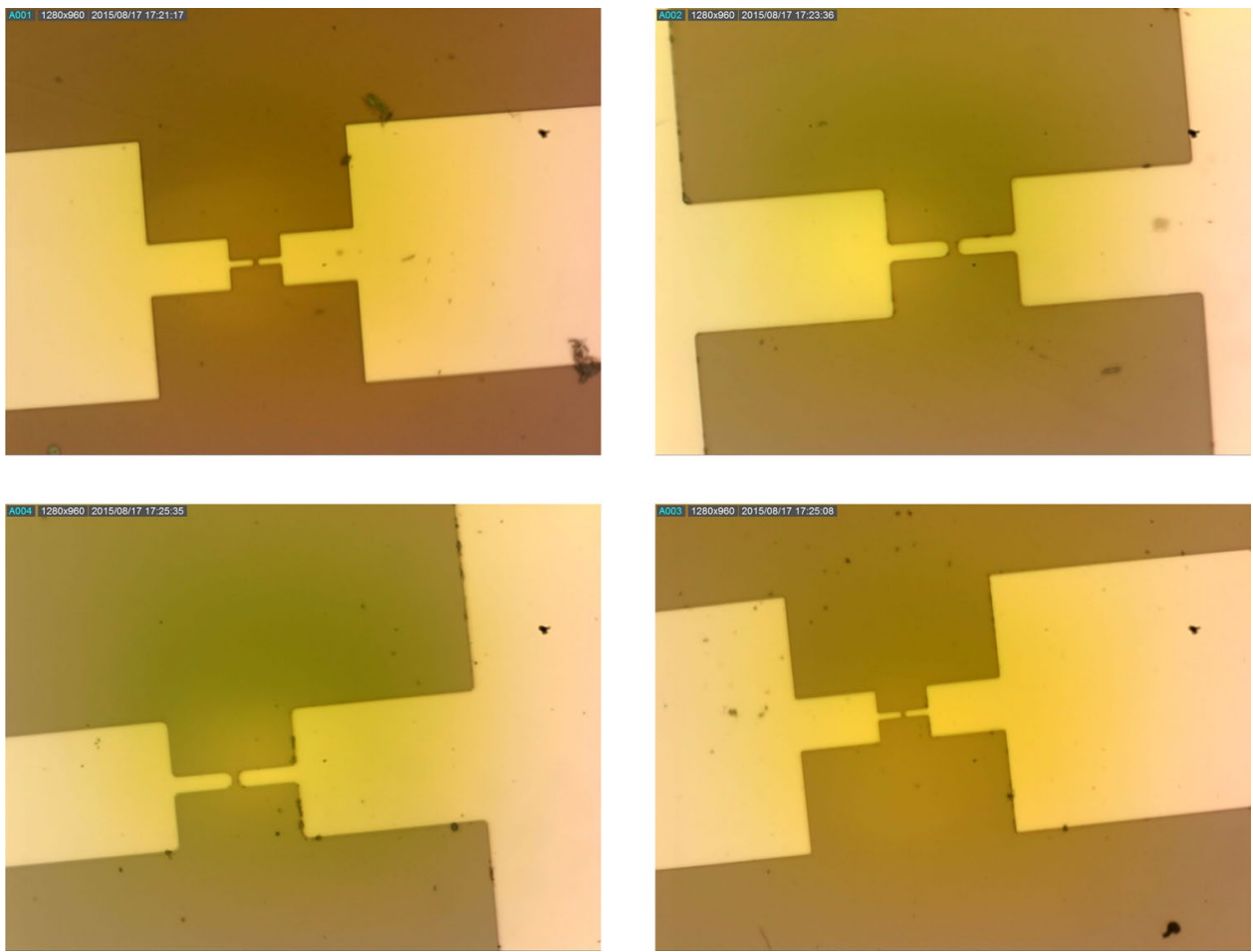

Fig. 5 The $I-V$ characteristics of the fabricated device with a gap between the electrodes of $1 \mu \mathrm{m}$ at a frequency of $7 \mathrm{MHz}$

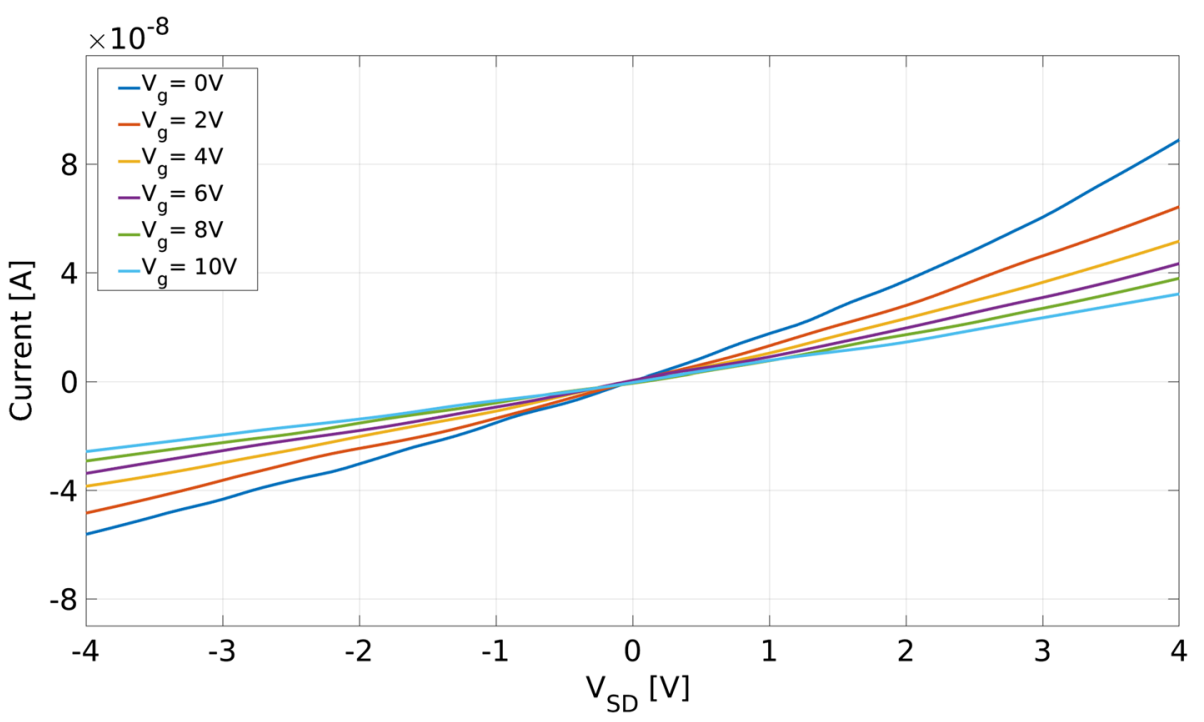

different fabricated devices with a gap between the electrodes of $2 \mu \mathrm{m}$ at a frequency of $50 \mathrm{KHz}$. Here, the deviation from linearity reveals that the aligned SWCNT(s) were semiconducting [41].

An important point to notice is that the model presented herein applies for a single SWCNT while the experimental results provide the $I-V$ characteristic of SWCNT bundles, so we see a back-gate response in the $I-V$ curves and very little deviation beginning at high source-drain voltages. In this frequency interval, bundles of metallic semiconductor SWCNTs with dominant metallic (low-bandgap semiconductor) behavior are aligned. In fact, the high linearity of the curves reveals that most of the deposited CNT(s) were metallic.

We now consider the linearity of the $I-V$ characteristics of the SWCNT-FET. In the case of a semiconductor, be it intrinsic, $p$-type, or $n$-type, in the absence of an external voltage, thermal agitation of electrons in the valance band results in the continuous creation of electron-hole pairs; i.e., electrons move from the valence to conduction band with a lifetime, resulting in a constant concentration of free electrons and holes at a constant temperature. When using a SWCNT as the channel in a CNT-FET, the shape of the 
Fig. 6 The $I-V$ characteristics of the fabricated device with a gap between the electrodes of $2 \mu \mathrm{m}$ at a frequency of $7 \mathrm{MHz}$

Fig. 7 The $I-V$ characteristics of the fabricated device with an electrode gap of $2 \mu \mathrm{m}$ at a frequency of $50 \mathrm{kHz}$
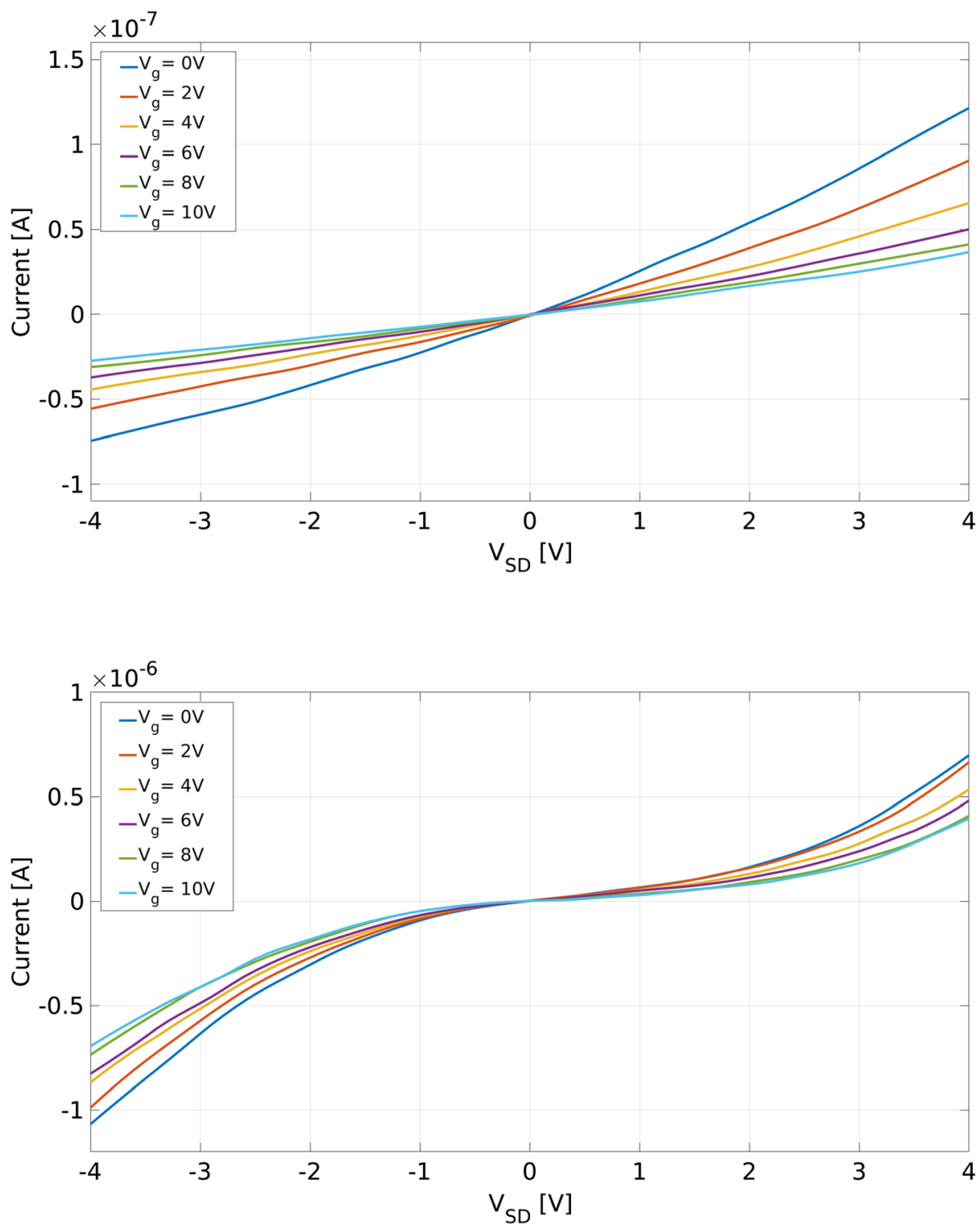

Fig. 8 The $I-V$ characteristics of a fabricated device with a gap between the electrodes of $2 \mu \mathrm{m}$ at a frequency of $50 \mathrm{KHz}$

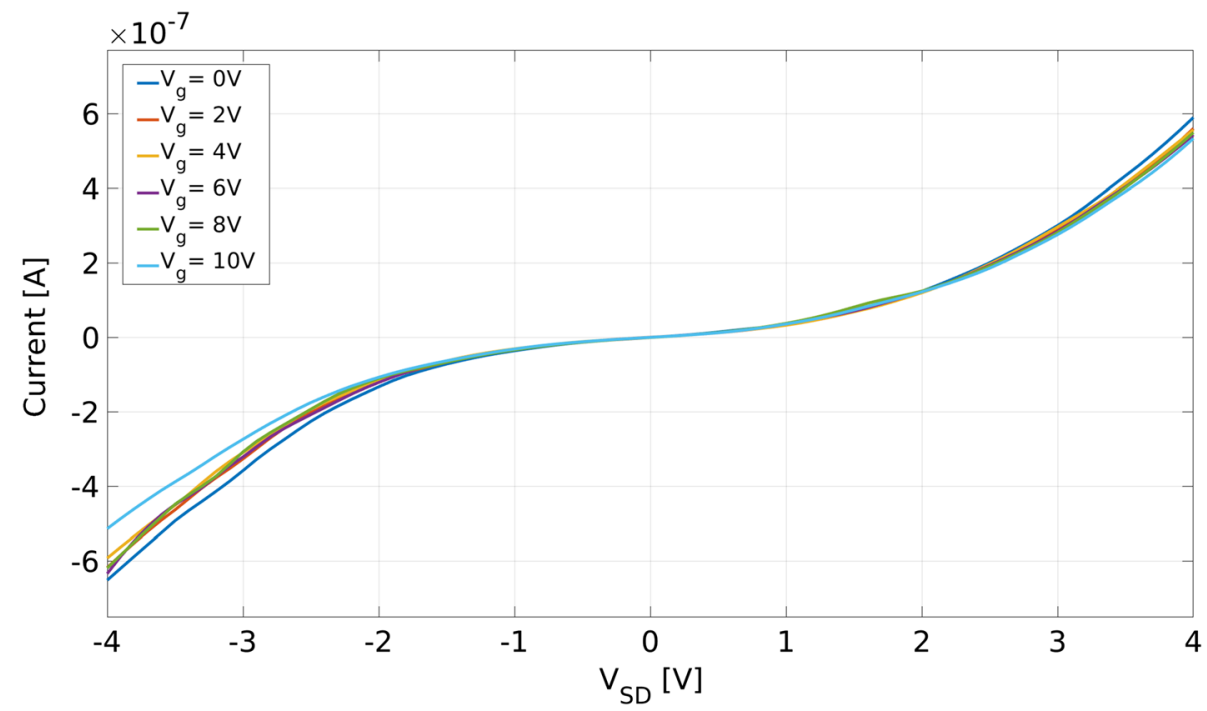


current-voltage characteristic is strongly affected by the potential profile across the channel [42]. Using a self-consistent field method, with increasing source-drain voltage more states become available for conduction and the electron (hole) density in the conduction band increases in the case of a semiconductor, so increasing the external voltage results in an increase in the concentration of electrons and holes (up to a point that is not shown on our curves) with a consequent continuous increase in the conductivity, which results in the deviation from linearity observed in the case of semiconductor SWCNTs [42].
The asymmetry apparent in these curves originates from the different ohmic contacts of the SWCNTs with metallic electrodes at the two endpoints. As shown in Fig. 9, when a symmetric voltage is applied, the resulting $I-V$ curve is not symmetric, which as stated above is because of the different contacts at the two endpoints (Figs. 10, 11, 12).

\subsection{SEM images}

Scanning electron microscopy images of some of the fabricated devices are now presented. On the pictures on the
Fig. 9 The $I-V$ characteristics of the fabricated device with a gap between the electrodes of $1 \mu \mathrm{m}$ at a frequency of $50 \mathrm{kHz}$ and $V_{\mathrm{g}}=0 \mathrm{~V}$. The asymmetry of the $I-V$ curve originates from the two different ohmic contacts
Fig. 10 Left: SEM images of the fabricated devices corresponding to the $I-V$ curve shown in Fig. 7. Right: a picture showing some ropes of carbon nanotubes between the two electrodes
Fig. 11 Left: SEM images of the fabricated device corresponding to the $I-V$ shown in Fig. 7. Right: a picture shows some ropes of carbon nanotubes between the two electrodes
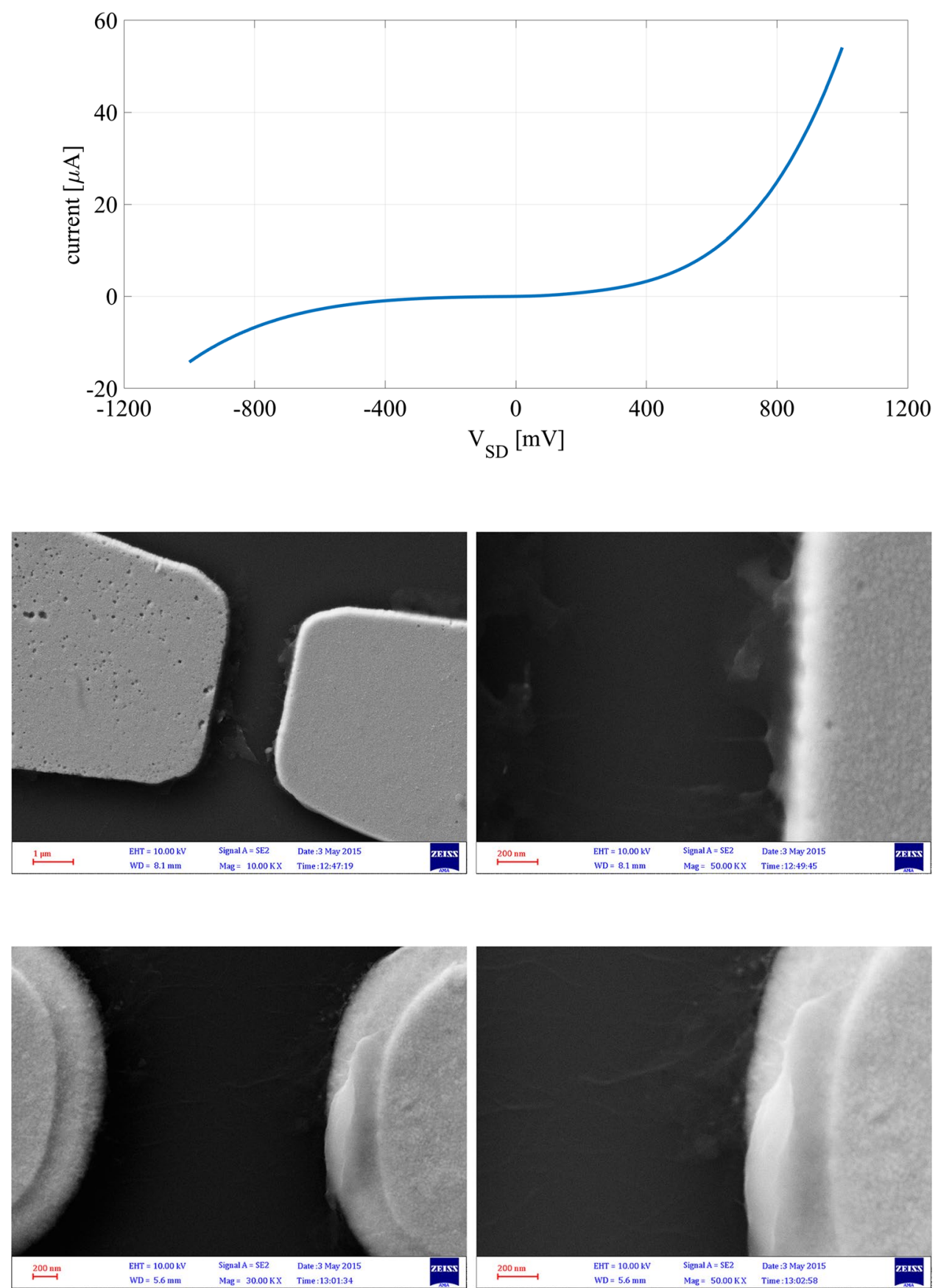
Fig. 12 Left: SEM images of the fabricated device corresponding to the $I-V$ shown in Fig. 9. Right: a picture showing some ropes of carbon nanotubes between the two electrodes
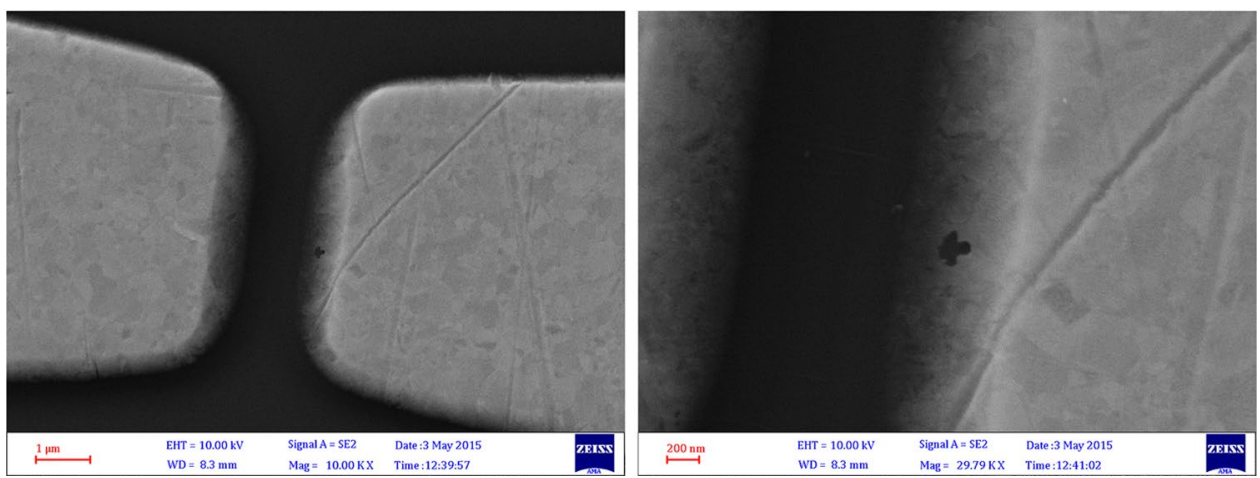

right, some ropes of carbon nanotubes consisting of one or more single-walled carbon nanotubes are apparent. Another point worth mentioning is the different currents at the same voltage in the $I-V$ characteristic curves of the fabricated devices, which seems to be because of the different numbers of SWCNTs aligned between the electrodes in the different devices.

\section{Conclusions}

Experimental results show that the correct frequency interval to achieve the alignment of single-walled carbon nanotubes via the DEP process can be identified by using the ellipsoid model, and in the case of large-gap semiconductor SWCNTs shows a great difference (two orders of magnitude) from that obtained using the spherical model that is prevalent in literature. Choosing the correct frequency interval as suggested by this theoretical model and adjusting the other parameters of the process such as the geometry of the electrodes and changing the metal electrodes can enable realization of more effective carbon nanotube field-effect transistors based on the DEP process. It is also clear that, when choosing the correct parameter values, dielectrophoresis can be applied as a reliable process for fabrication of carbon nanotube FETs on a large scale.

Acknowledgements Open access funding provided by Austrian Science Fund (FWF). A.K. and C.H. acknowledge financial support given by the FWF (Austrian Science Fund) START Project No. Y660 (PDE Models for Nanotechnology).

Open Access This article is licensed under a Creative Commons Attribution 4.0 International License, which permits use, sharing, adaptation, distribution and reproduction in any medium or format, as long as you give appropriate credit to the original author(s) and the source, provide a link to the Creative Commons licence, and indicate if changes were made. The images or other third party material in this article are included in the article's Creative Commons licence, unless indicated otherwise in a credit line to the material. If material is not included in the article's Creative Commons licence and your intended use is not permitted by statutory regulation or exceeds the permitted use, you will need to obtain permission directly from the copyright holder. To view a copy of this licence, visit http://creativecommons.org/licenses/by/4.0/.

\section{References}

1. Kalantari-Nejad, R., Bahrami, M., Rafii-Tabar, H., Rungger, I., Sanvito, S.: Computational modeling of a carbon nanotube-based DNA nanosensor. Nanotechnology 21(44), 445501 (2010)

2. Zhang, T., Mubeen, S., Myung, N.V., Deshusses, M.A.: Recent progress in carbon nanotube-based gas sensors. Nanotechnology 19(33), 332001 (2008)

3. Sinha, N., Ma, J., Yeow, J.T.: Carbon nanotube-based sensors. J. Nanosci. Nanotechnol. 6(3), 573-590 (2006)

4. Auvray, S., Derycke, V., Goffman, M., Filoramo, A., Jost, O., Bourgoin, J.-P.: Chemical optimization of self-assembled carbon nanotube transistors. Nano Lett. 5(3), 451-455 (2005)

5. Keren, K., Berman, R.S., Buchstab, E., Sivan, U., Braun, E.: DNA-templated carbon nanotube field-effect transistor. Science 302(5649), 1380-1382 (2003)

6. Yu, G., Cao, A., Lieber, C.M.: Large-area blown bubble films of aligned nanowires and carbon nanotubes. Nat. Nanotechnol. 2(6), 372 (2007)

7. Li, X., Zhang, L., Wang, X., Shimoyama, I., Sun, X., Seo, W.-S., Dai, H.: Langmuir-Blodgett assembly of densely aligned singlewalled carbon nanotubes from bulk materials. J. Am. Chem. Soc. 129(16), 4890-4891 (2007)

8. Jin, S., Whang, D., McAlpine, M.C., Friedman, R.S., Wu, Y., Lieber, C.M.: Scalable interconnection and integration of nanowire devices without registration. Nano Lett. 4(5), 915-919 (2004)

9. Engel, M., Small, J.P., Steiner, M., Freitag, M., Green, A.A., Hersam, M.C., Avouris, P.: Thin film nanotube transistors based on self-assembled, aligned, semiconducting carbon nanotube arrays. ACS Nano 2(12), 2445-2452 (2008)

10. Pohl, H.A.: The motion and precipitation of suspensoids in divergent electric fields. J. Appl. Phys. 22(7), 869-871 (1951)

11. Vijayaraghavan, A., Blatt, S., Weissenberger, D., Oron-Carl, M., Hennrich, F., Gerthsen, D., Hahn, H., Krupke, R.: Ultra-largescale directed assembly of single-walled carbon nanotube devices. Nano Lett. 7(6), 1556-1560 (2007)

12. Stokes, P., Khondaker, S.I.: High quality solution processed carbon nanotube transistors assembled by dielectrophoresis. Appl. Phys. Lett. 96(8), 083110 (2010)

13. An, L., Friedrich, C.: Dielectrophoretic assembly of carbon nanotubes and stability analysis. Prog. Nat. Sci. Mater. Int. 23(4), 367-373 (2013)

14. Wu, P.-F., Lee, G.-B.: Assembly of carbon nanotubes between electrodes by utilizing optically induced dielectrophoresis and dielectrophoresis. Adv. OptoElectron. (2011) 
15. Vijayaraghavan, A.: Bottom-up assembly of nano-carbon devices by dielectrophoresis. Phys. Status Solidi (b) 250(12), 2505-2517 (2013)

16. Abdulhameed, A., Abdul Halin, I., Mohtar, M. N., Hamidon, M. N.: The role of medium on the assembly of carbon nanotube by dielectrophoresis. J. Dispers. Sci. Technol. 1-12 (2019)

17. Xi, N., Lai, K.: Nano Optoelectronic Sensors and Devices: Nanophotonics from Design to Manufacturing. William Andrew (2011)

18. Abbaszade, M., Mohebbi, M.: Fourth-order numerical solution of a fractional PDE with the nonlinear source term in the electroanalytical chemistry. Iran. J. Math. Chem. 3(2), 195-220 (2012)

19. Abbaszadeh, M.: Error estimate of second-order finite difference scheme for solving the Riesz space distributed-order diffusion equation. Appl. Math. Lett. 88, 179-185 (2019)

20. Dehghan, M., Abbaszadeh, M.: Error analysis and numerical simulation of magnetohydrodynamics (MHD) equation based on the interpolating element free galerkin (IEFG) method. Appl. Numer. Math. 137, 252-273 (2019)

21. Dehghan, M., Abbaszadeh, M.: An efficient technique based on finite difference/finite element method for solution of two-dimensional space/multi-time fractional Bloch-Torrey equations. Appl. Numer. Math. 131, 190-206 (2018)

22. Dehghan, M.: Finite difference procedures for solving a problem arising in modeling and design of certain optoelectronic devices. Math. Comput. Simul. 71(1), 16-30 (2006)

23. Dehghan, M., Abbaszadeh, M., Mohebbi, A.: Legendre spectral element method for solving time fractional modified anomalous sub-diffusion equation. Appl. Math. Model. 40(5-6), 3635-3654 (2016)

24. Khodadadian, A., Parvizi, M., Abbaszadeh, M., Dehghan, M., Heitzinger, C.: A multilevel Monte Carlo finite element method for the stochastic Cahn-Hilliard-Cook equation. Comput. Mech. 64(4), 937-949 (2019)

25. Dehghan, M.: On the solution of an initial-boundary value problem that combines neumann and integral condition for the wave equation. Numer. Methods Partial Differ. Equ. 21(1), 24-40 (2005)

26. Mohammadi, V., Dehghan, M., Khodadadian, A., Wick, T.: Numerical investigation on the transport equation in spherical coordinates via generalized moving least squares and moving kriging least squares approximations. Eng. Comput. (2019). https ://doi.org/10.1007/s00366-019-00881-3

27. Khodadadian, A., Heitzinger, C.: Basis adaptation for the stochastic nonlinear Poisson-Boltzmann equation. J. Comput. Electron. 15(4), 1393-1406 (2016)

28. Mirsian, S., Khodadadian, A., Hedayati, M., Manzour-ol Ajdad, A., Kalantarinejad, R., Heitzinger, C.: A new method for selective functionalization of silicon nanowire sensors and Bayesian inversion for its parameters. Biosens. Bioelectron. 142, 111527 (2019)
29. Khodadadian, A., Hosseini, K., Manzour-ol Ajdad, A., Hedayati, M., Kalantarinejad, R., Heitzinger, C.: Optimal design of nanowire field-effect troponin sensors. Comput. Biol. Med. 87, 46-56 (2017)

30. Khodadadian, A., Taghizadeh, L., Heitzinger, C.: Three-dimensional optimal multi-level Monte-Carlo approximation of the stochastic drift-diffusion-Poisson system in nanoscale devices. J. Comput. Electron. 17(1), 76-89 (2018)

31. Khodadadian, A., Stadlbauer, B., Heitzinger, C.: Bayesian inversion for nanowire field-effect sensors. J. Comput. Electron. 19(1), $147-159$ (2020)

32. Khodadadian, A., Taghizadeh, L., Heitzinger, C.: Optimal multilevel randomized quasi-Monte-Carlo method for the stochastic drift-diffusion-Poisson system. Comput. Methods Appl. Mech. Eng. 329, 480-497 (2018)

33. Khodadadian, A., Parvizi, M., Heitzinger, C.: An adaptive multilevel Monte Carlo algorithm for the stochastic drift-diffusionPoisson system. Comput. Methods Appl. Mech. Eng. 368, 113163 (2020)

34. Khodadadian, A., Heitzinger, C.: A transport equation for confined structures applied to the OprP, Gramicidin A, and KcsA channels. J. Comput. Electron. 14(2), 524-532 (2015)

35. Nayfeh, M.H., Brussel, M.K.: Electricity and Magnetism. Courier Dover, Mineola (2015)

36. Pethig, R.R.: Dielectrophoresis: theory, methodology and biological applications. Wiley, Hoboken (2017)

37. Gimsa, J.: A comprehensive approach to electro-orientation, electrodeformation, dielectrophoresis, and electrorotation of ellipsoidal particles and biological cells. Bioelectrochemistry 54(1), 23-31 (2001)

38. Landau, L.D., Bell, J., Kearsley, M., Pitaevskii, L., Lifshitz, E., Sykes, J.: Electrodynamics of Continuous Media, vol. 8. Pergamon, Oxford (2013)

39. Kozinsky, B., Marzari, N.: Static dielectric properties of carbon nanotubes from first principles. Phys. Rev. Lett. 96(16), 166801 (2006)

40. Xue, W., Li, P.: Dielectrophoretic deposition and alignment of carbon nanotubes. In: Carbon Nanotubes-Synthesis, Characterization Applications, pp. 171-190 (2011)

41. Collins, P.G., Bando, H., Zettl, A.: Nanoscale electronic devices on carbon nanotubes. Nanotechnology 9(3), 153 (1998)

42. Datta, S.: Quantum Transport: Atom to Transistor. Cambridge University Press, Cambridge (2005)

Publisher's Note Springer Nature remains neutral with regard to jurisdictional claims in published maps and institutional affiliations. 